Pacific Journal of Mathematic 


\section{TWO CHARACTERIZATIONS OF QUASI-FROBENIUS RINGS}

Edgar A. Rutter, JR.

The purpose of this paper is to characterize quasi-Frobenius, $Q F$, rings in terms of relationships assumed to exist for each cyclic or finitely generated left module between the module and its second dual, where duality is with respect to the ring. More specifically we prove that a left perfect ring is $Q F$ if every cyclic left module is reflexive or every finitely generated left module is (isomorphic to) a submodule of a free module. For rings with minimum condition on left or right ideals this later condition is equivalent to every finitely generated left module being torsionless or to the ring being a cogenerator in the category of finitely generated left modules. If annihilator relations are defined by means of the natural pairing between a module and its dual, this condition is also equivalent to every submodule of a finitely generated left module being an annulet.

One of Nakayama's [14] original characterizations of $Q F$ rings was as rings with minimum condition on left or right ideals in which every left ideal is a left annulet and every right ideal is a right annulet. Ikeda and Nakayama [7] proved that a finite dimensional algebra in which every left ideal is a left annulet is $Q F$ but Nakayama [15] gave an example which shows that this weaker assumption does not characterize $Q F$ rings even when one assumes minimum condition on both left and right ideals. For an arbitrary ring every left ideal being a left annulet is easily seen to be equivalent to every cyclic left module being torsionless. Thus our results may be regarded as an attempt to extend the one sided result of Ikeda and Nakayama mentioned above by making weaker finiteness assumptions and stronger assumptions about the relation of the members of various classes of left modules to their second duals. Our results are also related to those of Morita, Kawada and Tachikawa [12] who proved that a ring with minimum condition is $Q F$ if every left module is a submodule of a free module and of Faith and Walker [5] who recently showed that this characterization is still valid without the assumption of minimum condition. Finally we call attention to the investigations of B.L. Osofsky [17] which we cannot adequately relate to the above results in a few words.

1. Preliminaries. Let $M$ and $N$ be modules over a ring $R$. The bimodule character of $R$ induces an $R$-module structure on $M^{*}=$ 
$\operatorname{Hom}_{R}(M, R)$, the dual of $M$, of the opposite hand from that of $M$ and an $R$-homomorphism $\alpha: M \rightarrow N$ gives rise to an $R$-homomorphism $\alpha^{*}: N^{*} \rightarrow M^{*}$ so that $*$ is a contravariant functor. There is a natural homomorphism $\sigma_{M}: M \rightarrow M^{* *}$ defined by

$$
\sigma_{M}(x)(f)=f(x) \text {, for all } x \in M \text { and } f \in M^{*},
$$

which gives a natural transformation from the identity functor to the functor $* *$ and $M$ is called torsionless (resp. reflexive) of $\sigma_{M}$ is a monomorphism (resp. isomorphism). A thorough discussion of these ideas and the basic properties of $*$ is contained in Chapter 5 of the monograph of Jans [9] or they are summarized more concisely in Part II of Bass' paper [1]. Other excellent references for this duality theory are Dieudonne [2] or Morita [11]. We shall draw freely upon the above sources and our notation and terminology will be as consistent as possible with that of Bass [1] and Jans [9]. In particular $R$ will always denote a ring with identity, $J$ the (Jacobson) radical of $R$ and all $R$-modules will be unitary.

Let $X$ be a subset of $R$. Then $l(X)=\{r \in R: r x=0$ for all $x \in X\}$ and $r(X)=\{r \in R: x r=0$ for all $x \in X\}$. Any left (resp. right) ideal of the form $l(X)$ (resp. $r(X)$ ) is a left (resp. right) annulet. $R$ is quasi-Frobenius, $Q F$, in case: (1) each right ideal is a right annulet; (2) each left ideal is a left annulet; and (3) $R$ has the minimum condition on left or right ideals. $Q F$ rings have numerous other charaterizations to which we shall refer as needed.

A module $M \subseteq N$ is small in $N$ if $N=M+K$ implies $K=N$ for any submodule $K$ of $N$. A projective module $P$ is called a projective cover of a module $L$ if there exists an epimorphism of $P$ onto $M$ whose kernel is small in $P$. Semi-perfect rings are those for which every finitely generated module has a projective cover. They are also characterized by the assumptions that $R / J$ satisfies the minimum condition on one sided ideals and idempotents can be lifted modulo $J$ (see [1]). $R$ is called left perfect if every left $R$-module has a projective cover. The equivalence of the following conditions was established by Bass [1]: (1) $R$ is left perfect, (2) $R / J$ satisfies the minimum condition on one sided ideals and every nonzero right $R$ module contains a simple right $R$-module, (3) a direct limit of projective left $R$-modules is projective, and (4) $R / J$ satisfies the minimum condition on one sided ideals and if $\left\{a_{i}: i=0,1, \cdots\right\} \subseteq J$, there is an $n$ such that $a_{0} a_{1} \cdots a_{n}=0$. Thus if $R$ satisfies the minimum condition on left or right ideals, $R$ is left perfect.

2. Rings whose cyclic modules are reflexive. If $C$ is a cyclic left (right) $R$-module, $C$ is isomorphic to $R / I$ where $I$ is a left (right) ideal of $R$ and $C$ is torsionless if and only if $I$ is a left (right) annulet. 
A right (left) ideal $I$ of $R$ satisfies condition $S$ if and only if every $R$-homomorphism of $I$ into $R$ is given by left (right) multiplication by an element of $R$. It is easy to verify that $I$ satisfies condition $S$ if and only if $\operatorname{Ext}_{R}^{1}(R / I, R)=0$ (see [9]).

Lemma 1. Let $I$ be a left ideal of $R$. Then $R / I$ is reflexive if and only if

(1) $l(r(I))=I$ and

(2) $r(I)$ satisfies condition $S$.

Proof. $(R / I)^{*}$ is isomorphic to $r(I)$ under the map $\delta^{-1}$ defined by $\delta^{-1}(f)=f(1+I)$ for all $f \in(R / I)^{*}$. It is clear that the map $\alpha: R / l(r(I) \rightarrow$ $r(I)^{*}$ which sends each $r+l(r(I))$ into left multiplication by $r$ is an $R$-monomorphism. Furthermore, $\alpha$ is an isomorphism if and only if $r(I)$ satisfies condition $S$. A straight forward verification shows that $\alpha \circ \eta=\delta^{*} \circ \sigma_{R / I}$, where $\eta$ is the natural projection of $R / I$ onto $R / l(r(I))$. If $R / I$ is reflexive, (1) holds since $\eta$ must be a monomorphism and (2) holds since $\alpha$ must be an epimorphism as $\delta^{*}$ and $\sigma_{R / I}$ are both isomorphisms. If (1) and (2) hold, $\eta, \alpha$, and $\delta^{*}$ are all isomorphisms and hence so is $\sigma_{R / I}$.

The next result is immediate from Lemma 1 (and its right hand analog) and the "injective test theorem" [9, p. 49].

THEOREM 1. Every cyclic left and every cyclic right $R$-module is reflexive if and only if ${ }_{R} R$ and $R_{R}$ are injective $R$-modules, every left ideal of $R$ is a left annulet, and every right ideal of $R$ is a right annulet.

The above theorem is not new. It can be obtained by specializing results of Morita [11, Lemma 2.1 and Th. 2.4]. Such a ring need not be $Q F$ although it must be semi-perfect (see Osofsky [17, Example 1, p. 378 and Th. 2, p. 380]).

Lemma 2. Let $R$ be a left perfect ring. If every cyclic left $R$-module is reflexive, then $R_{R}$ is an injective right $R$-module.

Proof. First we show that if $S$ is any simple right $R$-module, $S^{*}$ is simple or zero. Since $S \cong R / I$ for some maximal right ideal $I, S^{*} \cong l(I)$. But $l(I)=0$ or is a minimal left ideal of $R$. Otherwise, there is a left ideal $0 \nsubseteq L \nsubseteq l(I)$. Then $I \leqq r(l(I)) \subseteq r(L) \leqq R$ and hence either $r(l(I))=r(L)$ or $r(L)=R$. But $L$ is an annulet so either $L=l(r(L))=l(r(l(I)))=l(I)$ or $L=l(r(L))=l(R)=0$. Since neither equality is possible we have reached a contradiction. 
Let $C_{1}, \cdots, C_{m}$ be a complete set of nonisomorphic simple left $R$-modules. Since $R$ is left perfect, each $C_{i}^{*}$ contains a simple (right) submodule $S_{i}$. The inclusion map $j$ of $S_{i}$ into $C_{i}^{*}$ induces a map $j^{*}: C_{i}^{* *} \rightarrow S_{i}^{*}$ which is nontrivial since $C_{i}^{*}$ is torsionless and is; therefore, an isomorphism since both $S_{i}^{*}$ and $C_{i}^{* *} \cong C_{i}$ are simple (left) $R$-modules. Hence $S_{1}, \cdots, S_{m}$ are distinct and so are a complete set of nonisomorphic simple right $R$-modules. Furthermore, they are torsionless and hence so is $R_{R} / J$ since it is completely reducible. Thus $J$ is a right annulet.

Now ${ }_{R} R / l(J)$ is reflexive by assumption and hence Lemma 1 implies that $r(l(J))=J$ satisfies condition $S$ or what is the same thing that $\operatorname{Ext}_{R}^{1}\left(R_{R} / J, R_{R}\right)=0$. Thus $R_{R}$ is an injective right $R$-module (see [10, Prop. 2.6, p. 251]).

THEOREM 2. The following statements are equivalent for any ring $R$.

(a) $R$ is quasi-Frobenius.

(b) $R$ is left perfect and every cyclic left $R$-module is reflexive.

Proof. That (a) implies (b) is well known (see [2] or [8]). Since $Q F$ rings are right self injective (see [3, Th. 18, pp. 11-12]) this implication is also immediate from Lemma 1. Assume (b). By Lemma $2, R_{R}$ is an injective right $R$-module and so [3, Th. 18, pp. 11-12] will imply that $R$ is $Q F$ if we show that $R$ has the minimum condition on left ideals. In view of [17, Lemma 11, p. 382], it suffices for this to show that ${ }_{R} J / J^{2}$ is finitely generated. Suppose it is not. Then there exists an exact sequence

$$
0 \rightarrow \bigoplus_{i=1}^{\infty} S_{i} \rightarrow{ }_{R} R / J^{2} \rightarrow{ }_{R} M \rightarrow 0
$$

where the $S_{i}$ are isomorphic simple modules and ${ }_{R} R / J^{2}$ and ${ }_{R} M$ are reflexive. Dualizing $\#$ gives an exact sequence $0 \rightarrow{ }_{R} M^{*} \rightarrow\left({ }_{R} R / J^{2}\right)^{*} \rightarrow \prod_{i=1}^{\infty} S_{i}^{*}$. Thus we have an exact sequence

$$
0 \rightarrow{ }_{R} M^{*} \rightarrow\left({ }_{R} R / J^{2}\right)^{*} \rightarrow \bigoplus_{a \in A} S_{a}^{*} \rightarrow 0,
$$

with each $S_{a}^{*} \cong S_{i}^{*}$, since $\prod_{i=1}^{\infty} S_{i}^{*}$ is completely reducible as each $S_{i}^{*}$ is simple (see the proof of Lemma 2). Since $R_{R}$ is an injective right $R$-module dualizing \#\# we obtain the exact sequence

$$
0 \rightarrow \prod_{a \in A} S_{A}^{* *} \rightarrow\left({ }_{R} R / J^{2}\right)^{* *} \rightarrow{ }_{R} M^{* *} \rightarrow 0 .
$$

Connecting $\#$ and $\# \#$ by means of the natural isomorphisms $\sigma_{R / J}$ and $\sigma_{M}$ one verifies easily that there is induced an $R$-isomorphism between $\bigoplus_{i=1}^{\infty} S_{i}$ and $\prod_{a \in A} S_{a}^{* *} \cong \prod_{a \in A} S_{a}$, with each $S_{a} \cong S_{1}$. 
Since the hypotheses are preserved under passage to the basic ring (see [6] or [16]), we may assume that $R$ is basic. Then $R / J$ is a ring direct sum of division rings so that $S_{1}$ is a one dimensional vector space over a division ring $D$. Thus $\prod_{a \in A} S_{a}$ and $\bigoplus_{i=i}^{\infty} S_{i}$ are isomorphic both as $R$-modules and as $D$-vector spaces. However; since $A$ is clearly infinite, $D$-dimension of $\prod_{a \in A} S_{a}=$ cardinality $\left(D^{A}\right)>\boldsymbol{\aleph}_{0}=$ $D$-dimension of $\bigoplus_{i=1}^{\infty} S_{i}$. This contradiction completes the proof.

CoROllary 1. Let $R$ be a ring with minimum condition on left or right ideals. If $C$ is isomorphic to $C^{* *}$ for each cyclic left $R$ module, $R$ is quasi-Frobenius.

Proof. Since duals are torsionless (see [9]), every left ideal of $R$ is a left annulet. Hence in either case $R$ has minimum condition of left ideals. Since $C \cong C^{* *}$ they have the same composition length and $\sigma_{C}$ being a monomorphism must be an isomorphism.

3. Torsionless modules and submodules of free modules. If $S$ is a set, we shall denote the cardinality of $S$ by $|S|$ and if $\alpha$ is an ordinal number, we shall denote the cardinality of any set of order type $\alpha$ by $|\alpha|$. If $M$ is an $R$-module, $\|M\|$ will denote the smallest cardinal number for which $M$ has a set of $R$-generators of this cardinal. If $\left\{M_{\alpha}, \Pi_{\alpha}^{\beta}\right\}_{\alpha, \beta \in D}$ is a directed system of $R$-modules and $R$-homomorphisms over a directed set $D$ then we shall then denote the direct limit of this system by $\lim _{D} M_{\alpha}$ (see [4] or [19]).

THEOREM 3. The following statements are equivalent.

(a) $R$ is a quasi-Frobenius ring.

(b) $R$ is a left perfect ring and every finitely generated left $R$-module is (isomorphic to) a submodule of a projective left $R$-module.

Proof. That (a) implies (b) is well known (see [5] or [12]). Assume (b). Then by [5, Corollary 5.6, p. 216] it suffices to show that every left $R$-module is a submodule of a projective $R$-module. Suppose this is not so. Then there exists a smallest cardinal $\tau$ for which there is a left $R$-module $M$ with $\|M\|=\tau$ which cannot be embedded in a projective module. Then $\tau$ must be an infinite cardinal. Let $G$ be a set of $R$-generators for $M$ with $|G|=\|M\|=\tau$. Let $\gamma$ be the smallest ordinal associated with the cardinal $\tau$. Since $\tau$ is an infinite cardinal $\gamma$ is a limit ordinal, i.e., $\gamma$ does not have an immediate predecessor. Furthermore, if $\alpha$ is any ordinal less than $\gamma$, then $|\alpha|<|\gamma|$. The set of all ordinals strictly less than $\gamma$ is a set of order type $\gamma$ and cardinality $|\gamma|=\tau$. Let $\alpha \rightarrow g_{\alpha}$ be a one-to-one correspondence between the set of all ordinals $<\gamma$ and $G$. For each 
ordinal $\alpha<\gamma$ define $M_{\alpha}$ to be the submodule of $M$ generated by $G_{\alpha}=\left\{g_{\beta}: \beta<\alpha\right\}$. Then $\left\{M_{\alpha}\right\}_{\alpha<\gamma}$ is a chain of submodules of $M$ and $M=\mathrm{U}_{\alpha<\gamma} M_{\alpha}$. Thus we may regard the $M_{\alpha}$ and their inclusion maps as a direct system having $M$ as direct limit. The proof will be completed by showing the existence of a direct system $\left\{P_{\alpha}, \prod_{\alpha}^{\beta}\right\}_{\alpha, \beta<\gamma}$ of projective modules and a morphism $\phi$ of direct systems such that

(1) for all $\alpha<\gamma, \phi_{\alpha}: M_{\alpha} \rightarrow P_{\alpha}$ is a monomorphism and

(2) for each $\alpha<\gamma$, if $|\alpha|$ is finite, $\left\|P_{\alpha}\right\|$ is finite and if $|\alpha|$ is infinite, $\left\|P_{\alpha}\right\| \leqq|\alpha|$.

For then we would have $\bar{\phi}=\underline{\lim } \phi_{\alpha}: \lim M_{\alpha}=M \rightarrow \underline{\lim P_{\alpha}=\bar{P}}$. This is a contradiction since $\bar{P}$ is projective $\overrightarrow{a s} R$ is left perfect and $\bar{\phi}$ is a monomorphism since each $\phi_{\alpha}$ is monic. Condition 2 is used only in proving the existence of the desired direct system.

We prove the existence of this direct system and of $\phi$ by transfinite induction. We take $P_{0}=0$ and $\phi^{0}=0$. Now to show that if $\phi^{\beta}$ and $P_{\beta}$ have been defined for all $0 \leqq \beta<\alpha<\gamma$, then $\phi^{\alpha}$ and $P_{\alpha}$ can be defined. We consider two cases.

Case I ( $\alpha$ not a limit ordinal). Define $X$ to be the submodule

$$
\left\{\left(\phi^{\alpha-1}(m),-m\right): m \in M_{\alpha-1}\right\}
$$

of $P_{\alpha-1} \oplus M_{\alpha}$ and $T=\left(P_{\alpha-1} \oplus M_{\alpha}\right) / X$ and let $f_{1}$ and $f_{2}$ be the $R$ homomorphisms from $P_{\alpha-1}$ and $M_{\alpha}$, respectively, into $T$ obtained by composing the usual injections into the direct sum with the natural projection onto the quotient module. It is straightforward to verify that $X$ was defined so that $f_{1}$ and $f_{2}$ are both monomorphisms and $f_{2}=f_{1} \circ \phi^{\alpha-1}$. Also $\|T\| \leqq\left\|P_{\alpha-1}\right\|+\left\|M_{\alpha}\right\| \leqq\left\|P_{\alpha-1}\right\|+|\alpha|$ which, in view of Condition 2, is finite if $|\alpha|$ is finite and $\leqq|\alpha-1|+|\alpha|=$ $|\alpha|<|\gamma|=\tau$ if $|\alpha|$ is infinite. Thus by the choice of $\tau$ there exists a projective module $P_{\alpha}$ containing $T$ and satisfying the requirement of condition (2). We can take $\phi^{\alpha}=i \circ f_{2}$, where, $i$ is the inclusion map of $T$ into $P_{\alpha}, \prod_{\alpha-1}^{\alpha}=i \circ f_{1}$ and $\prod_{\beta}^{\alpha}=\prod_{\alpha-1}^{\alpha} \circ \prod_{\beta}^{\alpha-1}$ if $\beta<\alpha$.

Case II ( $\alpha$ a limit ordinal). Since $M_{\alpha}=\mathrm{U}_{\beta<\alpha} M_{\beta}$, it is the direct limit of these modules. Thus we may take $P_{\alpha}=\lim _{\beta<\alpha} P_{\beta}, \phi^{\alpha}=\lim _{\beta<\alpha} \phi^{\beta}$, and $\Pi_{\beta}^{\alpha}$ to be the natural projection into the direct limit. It remains only to observe that $\left\|P_{\alpha}\right\| \leqq|\alpha|$. But for each $\beta<\alpha, P_{\beta}$ has a set of generators $B_{\beta}$ with $\left|B_{\beta}\right| \leqq|\alpha|$ and so $P_{\alpha}$ is generated by $B=$ $\mathrm{U}_{\beta<\alpha} \Pi_{\beta}^{\alpha}\left(B_{\beta}\right)$ and $|B| \leqq|\alpha||\alpha|=|\alpha|$.

Nakayama ]15, p. 48] showed how to construct a ring with precisely three left ideals $R \nsupseteq J \nsupseteq 0$ which also has minimum condition on right ideals but isn't $Q F$. There also exists a ring with the same 
three left ideals which doesn't even have the minimum condition on right ideals (see [18]). For either of these rings $R, O$, and $J=R / J$ are the only cyclic left modules so both of these rings have the property that every cyclic left $R$-module is contained in a free $R$ module, in fact, is contained in $R$. On the other hand Faith and Walker [5, Corollary 5.10, p. 217] have shown that if every cyclic left and every cyclic right $R$-module is contained in a projective $R$ module, $R$ is $Q F$.

COROLlaRY 2. Let $R$ be a ring with minimum condition on left (or right) ideals. Then the following statements are equivalent.

(a) $R$ is quasi-Frobenius.

(b) ${ }_{R} R$ is a cogenerator for the category of finitely generated left $R$-modules.

(c) Every finitely generated left $R$-module is torsionless.

Proof. That (a) implies (b) is well known (see [5, Corollary 5.4, p. 216]) and the equivalence of (b) and (c) is immediate from the definitions. Assume (c) and let ${ }_{R} M$ be finitely generated over $R$. Then $0=\cap \operatorname{ker} f, f \in M^{*}$. Thus there exist $f_{1}, \cdots, f_{n} \in M^{*}$ such that $\bigcap_{i=1}^{n} \operatorname{ker} f_{i}=0$. Hence there is a monomorphism of $M$ into the free module $\bigoplus_{i=1}^{n} R_{i}$, where $R_{i} \cong R$ for $i=1, \cdots, n$, defined by $m \rightarrow\left(f_{1}(m)\right.$, $\left.\cdots, f_{n}(m)\right)$. Thus Theorem 3 implies $R$ is $Q F$.

Osofsky [17, Example 1, p. 378] showed that even if $R^{R}$ is an injective cogenerator $R$ need not be $Q F$ but it is an open question whether or not a left perfect ring $R$ such that $R^{R}$ is an injective cogenerator is $Q F$.

The author wishes to thank his advisor, Professor T.J. Head, for his aid and encouragement. The author also wishes to thank the referee who extended our original version of Theorem 2 from rings with minimum condition to left perfect rings and generously allowed us to include his result in this paper.

\section{REFERENCES}

1. H. Bass, Finitistic dimension and a homological generalization of semi-primary rings, Trans. Amer. Math. Soc. 95 (1960), 466-488.

2. J. Dieudonne, Remarks on quasi-Frobenius rings, Illinois J. Math. 2 (1958), 346-354.

3. S. Eilenberg, and T. Nakayama, On the dimension of modules and algebras, II, Frobenius algebras and quasi-Frobenius rings, Nagoya Math. J. 9 (1955), 1-16.

4. S. Eilenberg and N. Steenrod, Foundations of algebraic topology, Princeton University Press, Princeton, N.J., 1952.

5. C. Faith and E. Walker, Direct sum representations of injective modules, J. Algebra 5 (1967), 203-221.

6. R. Gordon, Rings faithfully represented on their left socle, J. Algebra 7 (1967), 303-342. 
7. M. Ikeda and T. Nakayama, Supplementary remarks on Frobeniusian algebras, II, Oska Math. J. 2 (1950), 7-12.

8. J. P. Jans, Duality in Noetherian rings, Proc. Amer. Math. Soc. 12 (1961), 829-835. 9. - Rings and homology, Holt, Rinehart and Winston, New York, 1964.

10. H. Y. Mochizuki, Finitistic global dimension of rings, Pacific J. Math. 15 (1965), 249-258.

11. K. Morita, Duality for modules and its application to the theory of rings with minimum condition, Sci. Rept. Tokyo Kyoiku Daiguku A6 (1958), 83-142.

12. K. Morita, Y. Kawada and H. Tachikawa, On injective modules Math. Z. 68 (1957), 217-226.

13. T. Nakayama, On Frobeniusian algebras, I, Ann. Math. 40 (1939), 611-633.

14. ——, On Frobeniusian algebras, II, Ann. Math. 42 (1941), 1-21.

15. - Supplementary remarks on Frobeniusian algebras, I, Proc. Japan Acad. 25 (1949), 45-50.

16. M. Osima, Notes on basic rings, Math. J. Okayama Univ. 2 (1953), 103-110.

17. B. L. Osofsky, A generalization of quasi-Frobenius rings, J. Algebra 4 (1966), 373-387.

18. A. Rosenberg, and D. Zelinski, Finiteness of the injective hull, Math. Z. 70 (1959), $372-380$.

19. R. G. Swan, The theory of sheaves, The Univ. of Chicago Press, Chicago, Illinois, 1964.

Received March 26, 1968. Portions of this paper are contained in the author's doctoral dissertation which was submitted to the graduate faculty of Iowa State University in 1965 .

The University OF KANSAS 


\section{PACIFIC JOURNAL OF MATHEMATICS}

\section{EDITORS}

H. ROYDEN

Stanford University

Stanford, California

\section{RichaRd PIERCE}

University of Washington Seattle, Washington 98105
J. DugundJI

Department of Mathematics

University of Southern California

Los Angeles, California 90007

BASIL GORDON

University of California

Los Angeles, California 90024

\section{ASSOCIATE EDITORS}
E. F. BECKENBACH
B. H. NeUMANN
F. WOLF
K. YOSHIDA

\section{SUPPORTING INSTITUTIONS}

UNIVERSITY OF BRITISH COLUMBIA CALIFORNIA INSTITUTE OF TECHNOLOGY

UNIVERSITY OF CALIFORNIA

MONTANA STATE UNIVERSITY

UNIVERSITY OF NEVADA

NEW MEXICO STATE UNIVERSITY

OREGON STATE UNIVERSITY

UNIVERSITY OF OREGON

OSAKA UNIVERSITY

UNIVERSITY OF SOUTHERN CALIFORNIA

\author{
STANFORD UNIVERSITY \\ UNIVERSITY OF TOKYO \\ UNIVERSITY OF UTAH \\ WASHINGTON STATE UNIVERSITY \\ UNIVERSITY OF WASHINGTON \\ AMERICAN MATHEMATICAL SOCIETY \\ CHEVRON RESEARCH CORPORATION \\ TRW SYSTEMS \\ NAVAL WEAPONS CENTER
}

The Supporting Institutions listed above contribute to the cost of publication of this Journal, but they are not owners or publishers and have no responsibility for its content or policies.

Mathematical papers intended for publication in the Pacific Journal of Mathematics should be in typed form or offset-reproduced, double spaced with large margins. Underline Greek letters in red, German in green, and script in blue. The first paragraph or two must be capable of being used separately as a synopsis of the entire paper. It should not contain references to the bibliography. Manuscripts, in duplicate if possible, may be sent to any one of the four editors. Please classify according to the scheme of Math. Rev. 36, 1539-1546. All other communications to the editors should be addressed to the managing editor, Richard Arens, University of California, Los Angeles, California, 90024.

50 reprints are provided free for each article; additional copies may be obtained at cost in multiples of 50 .

The Pacific Journal of Mathematics is published monthly. Effective with Volume 16 the price per volume (3 numbers) is $\$ 8.00$; single issues, $\$ 3.00$. Special price for current issues to individual faculty members of supporting institutions and to individual members of the American Mathematical Society: $\$ 4.00$ per volume; single issues $\$ 1.50$. Back numbers are available.

Subscriptions, orders for back numbers, and changes of address should be sent to Pacific Journal of Mathematics, 103 Highland Boulevard, Berkeley, California, 94708.

PUBLISHED BY PACIFIC JOURNAL OF MATHEMATICS, A NON-PROFIT CORPORATION

Printed at Kokusai Bunken Insatsusha (International Academic Printing Co., Ltd.), 7-17. Fujimi 2-chome, Chiyoda-ku, Tokyo, Japan. 


\section{Pacific Journal of Mathematics}

\section{Vol. 30, No. $3 \quad$ November, 1969}

Willard Ellis Baxter, Topological rings with property $(Y) \ldots \ldots \ldots \ldots . \ldots 5$

Sterling K. Berberian, Note on some spectral inequalities of $C . R$.

Putnam ..................................... 573

David Theodore Brown, Galois theory for Banach algebras . ........... 577

Dennis K. Burke and R. A. Stoltenberg, A note on p-spaces and Moore spaces ........................................ 601

Rafael Van Severen Chacon and Stephen Allan McGrath, Estimates of positive contractions....................................... 609

Rene Felix Dennemeyer, Conjugate surfaces for multiple integral problems in the calculus of variations ........................... 621

Edwin O. Elliott, Measures on countable product spaces.............. 639

John Moss Grover, Covering groups of groups of Lie type .............. 645

Charles Lemuel Hagopian, Concerning semi-local-connectedness and cutting in nonlocally connected continua .................. 657

Velmer B. Headley, A monotonicity principle for eigenvalues ........... 663

John Joseph Hutchinson, Intrinsic extensions of rings . . . . . . . . . . . . . 669

Harold H. Johnson, Determination of hyperbolicity by partial

prolongations .................................. 679

Tilla Weinstein, Holomorphic quadratic differentials on surfaces in $E^{3} \ldots 697$

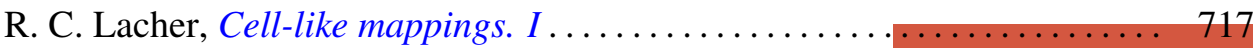

Roger McCann, A classification of centers

Curtis L. Outlaw, Mean value iteration of nonexpansive mappings in a

Banach space...

Allan C. Peterson, Distribution of zeros of solutions of a fourth order

differential equation.

Bhalchandra B. Phadke, Polyhedron inequality and strict convexity .. 765 Jack Wyndall Rogers Jr., On universal tree-like continua .

Edgar Andrews Rutter, Two characterizations of quasi-Frobenius rings

G. Sankaranarayanan and C. Suyambulingom, Some renewal theorems concerning a sequence of correlated random variables...

Joel E. Schneider, A note on the theory of primes........ . .

Richard Peter Stanley, Zero square rings .................

Edward D. Tymchatyn, The 2-cell as a partially ordered space

Craig A. Wood, On general Z.P.I.-rings................ 